Discussion Paper 135

Institute for Empirical Macroeconomics

Federal Reserve Bank of Minneapolis

90 Hennepin Avenue

Minneapolis, Minnesota 55480-0291

June 2000

\title{
Knowledge Exchange, Matching, and Agglomeration
}

\author{
Marcus Berliant \\ Washington University \\ Robert R. Reed III \\ Iowa State University \\ Ping Wang \\ Vanderbilt University
}

\begin{abstract}
*We thank Beth Allen, Kalyan Chatterjee, Ed Coulson, Robert Helsley, Bill Hoyt, Pat Kehoe, Derek Laing, Victor Li, Chris Phelan, Will Strange, and participants at the Midwest Mathematical Economics Conference at Northwestern University, the Regional Science Association International Meetings in Buffalo, the Society for Economic Dynamics Conference in Philadelphia and seminar participants at Academia Sinica, the Graduate School of Industrial Administration at Carnegie Mellon University, the Federal Reserve Bank of Minneapolis, the University of Kansas, the University of Kentucky, and Washington University in St. Louis for valuable comments and suggestions. The first author acknowledges financial support from the NSF grant No. SBR-9523940. The views expressed herein are those of the author(s) and not necessarily those of the Federal Reserve Bank of Minneapolis or the Federal Reserve System.
\end{abstract}




\begin{abstract}
Despite wide recognition of their significant role in explaining sustained growth and economic development, uncompensated knowledge spillovers have not yet been fully modeled with a microeconomic foundation. The main purpose of this paper is to illustrate the exchange of knowledge as well as its consequences on agglomerative activity in a general-equilibrium search-theoretic framework. Agents, possessing differentiated types of knowledge, search for partners to exchange ideas and create new knowledge in order to improve production efficacy. When individuals' types of knowledge are too diverse, a match is less likely to generate significant innovations. We demonstrate the extent of agglomeration has significant implications for the patterns of information flows in economies. Further, by simultaneously determining the patterns of knowledge exchange and the spatial agglomeration of an economy we identify additional channels for interaction between agglomerative activity and knowledge exchange. Finally, contrary to previous work in spatial agglomeration, our model suggests that agglomerative environments may be either under-specialized and under-populated or over-specialized and over-populated relative to the social optimum.
\end{abstract}




\section{Introduction}

Uncompensated knowledge spillovers have played a central role in explaining sustained growth and economic development. In their pioneering work, Romer (1986) and Lucas (1988) develop models in which the positive external effects of society's aggregate knowledge or human capital stock promote economic growth. The incorporation of this type of positive externality has resulted in abundant research in the areas of growth and development. These insights, however, raise many important but unsettled questions. How do knowledge spillovers occur? What are the consequences of knowledge spillovers on the advancement and concentration of economic activity? Lucas points out that interaction among economic agents is the key for the development of knowledge: “...human capital accumulation is a social activity involving groups of people...” (p. 19). Given that interaction seems to promote both knowledge acquisition and creation, various types of economic clusters may emerge as economic organizations to foster the transmission of information. The present paper is devoted to examining these important but largely open issues.

Marshall (1890), Kuznets (1962), and Jacobs (1969) stress that knowledge spillovers are the primary force for agglomeration, such as city formation, firm clustering, and geographical concentration of research activity. Marshall relates these benefits of agglomeration as follows:

"When an industry has chosen a locality for itself, it is likely to stay there long; so great are the advantages which people following the same skilled trade get from near neighborhood to one another. The mysteries of the trade become no mysteries; but are as it were in the air, and children learn many of them unconsciously. Good work is appreciated, inventions and improvements in machinery, in processes and the general organization of the business have their merits promptly discussed; if one man starts a new idea, it is taken up by others and combined with suggestions of their own; and thus it becomes the source of new ideas." (p. 352)

Kuznets echoes Marshall's views:

"Creative effort flourishes in a dense intellectual atmosphere, and it is hardly accident that the locus of intellectual progress (including that of the arts) lies in the larger cities, not in the bucolic surroundings of the thinly settled countryside ... the possibility of more intensive intellectual contact ... afforded by greater numbers may be an important factor in stepping up the rate of additions to new knowledge." (pp. 328-9) 
These arguments suggest that agglomeration promotes the transmission of knowledge due to lower costs of communication in dense environments. ${ }^{1}$ Yet, despite the clearly important role of geography for the propagation of knowledge, spatial considerations have received limited attention in the theoretical literature. ${ }^{2}$ We attempt to fill this gap by developing a simple search-theoretic model particularly suitable for analyzing the knowledge creation mechanism and its interactions with agglomerative activity. We believe the random-matching model to be the most appropriate for studying these issues because it provides an explicit notion of transactions costs (search and entry frictions) and patterns of interaction (knowledge exchange). The latter aspect, in particular, allows us to analyze the relationships between endogenous knowledge exchange and endogenous population agglomeration.

In our economy, agents, such as individual consumers/workers, firms and patent holders, possess horizontally differentiated types of knowledge and search for partners to exchange ideas and create new knowledge, so as to improve production efficacy. We consider that heterogeneity (in terms of different types of knowledge) plays a role in the acquisition and advance of knowledge. When individuals' types of knowledge are too diverse, a match is less likely to generate significant innovations. The same applies when individuals' types are too similar, so little is added through collaboration. We first endogenously

${ }^{1}$ Empirical studies seem to concur with these insights by demonstrating that cities provide the opportunity for knowledge transmission to occur. For example, Jaffe et al $(1993,1996)$ show that knowledge spillovers are localized in the sense that patents are more likely to cite previous patents from the same area and spillovers can cross national borders only with delay. In particular, they show inventors from the United States are more likely to cite previous U.S. patents. Canadian inventors are more likely to cite previous U.S. patents than European and Japanese inventors. They also obtain that localization effects diminish over time. Additional work by Glaeser et al (1992) and Henderson et al (1995) finds that spillovers occur both within and between industries and characteristics of urban areas play a role in the location decisions of industries.

${ }^{2}$ Glaeser (1997) considers the role of cities for the propagation of knowledge. His focus, however, is more on the role of cities to promote knowledge acquisition by younger, less skilled workers from older and more skilled workers. In contrast, we focus on the potential to learn from individuals with different types of knowledge or ideas as a stimulus for the evolution of knowledge and agglomeration. Helsley and Strange (1999) provide an alternative view on cities and innovative activity. They describe how agglomeration facilitates innovation by lowering the costs of innovation through a higher density of factor suppliers. 
determine the range of agents with whom an individual will undertake knowledge exchange, hereafter called the knowledge spread, and characterize its determinants in a closed-city model. We later consider the case of a small, open city. This allows us to study the interactions between the determinants of knowledge exchange and the endogenous process of population agglomeration. By characterizing the role of heterogeneity for knowledge creation, our structure provides insights into questions regarding human capital accumulation, the patterns of information flows, and their interactions with agglomerative activity.

In our first model, we consider the case of a closed-city where the population size is fixed. ${ }^{3}$ This exercise is important for two reasons. First, it is valuable in the endogenous growth context as we provide explanations behind the determinants of knowledge exchange. We show that economies with higher search or market frictions will have more diversified patterns of information exchange so that individuals obtain more, but generally less effective, interactions with others. Thus, market frictions play an important role in the patterns of human capital accumulation and an economy's rate of growth. ${ }^{4}$ Second, we show that the extent of agglomeration may influence an economy's pattern of information flows and development. We demonstrate that economies with a higher population size will have more specialized patterns of knowledge exchange and higher welfare. ${ }^{5}$

In our second model, we simultaneously determine the patterns of knowledge exchange and the spatial agglomeration of the economy. Extending our analysis to determine the economy's population size provides additional channels for interaction between agglomerative activity and knowledge exchange.

Within the closed-city framework, the level of technology does not affect the range of individuals agents

${ }^{3}$ For a discussion of the properties and uses of the classical closed and open city models of urban economics, see Fujita (1989).

${ }^{4}$ For papers examining the links between market frictions and economic growth, see Aghion and Howitt (1994), Laing, Palivos, and Wang (1995), and Acemoglu (1996).

${ }^{5}$ Pred (1966), for example, argues that "[i]t is logical that the larger the city, the larger the number of intentionally and unintentionally overlapping information fields of laborers and other industrial personnel, the larger the volume of influential short-distance information flows..." (pp. 128-9). 
try to meet. Under the endogenous population migration setup, however, a higher level of technology improves production efficacy and further encourages agglomeration. With a higher population size, individuals can concentrate on more effective knowledge exchange and thus try to work with a smaller range of individuals.

Contrary to previous work in spatial agglomeration, we demonstrate the possibility that agglomerative environments may be under-specialized and underpopulated, or alternatively overspecialized and over-populated relative to the social optimum. This occurs for two reasons. First, as individuals decide to migrate, they do not take into account the effect of their entry on the total population mass. This is a congestion externality which is common in the urban economics literature. Second, we provide a channel for agglomeration inefficiencies to arise due to inefficiencies associated with knowledge exchange. This results from a matching externality - since the arrival rate of potential collaborators may depend on the remaining mass of unmatched individuals, agents do not take into account the effect of their choices on the pool of potential collaborators and the probability of matching for other individuals. This establishes an additional reason for knowledge transfer and innovation to be socially inefficient. ${ }^{6}$ Because of the matching externality, the decentralized equilibrium may be under-specialized and under-populated relative to the social optimum.

There are some related papers in regard to an urban economics context. Fujita and Ogawa (1982) study urban configurations by constructing a "locational potential function" to account for the externality of business agglomeration. In their model, they postulate that firms' profits are lower when firms are located farther apart. Berliant, Peng and Wang (1998) examine urban structures in the presence of uncompensated inter-firm knowledge spillovers which decrease with the distance between firms. Palivos

${ }^{6}$ For example, Romer (1986) and Lucas (1988) show that knowledge accumulation may be below the socially optimal level because individuals do not take into account the benefits of their own level of human capital on aggregate learning. In contrast, we show that human capital accumulation may be inefficient because of the economy's matching externality. 
and Wang (1997) consider the uncompensated knowledge spillover as the primary force generating sustained growth to determine the optimal paths for output and population growth in a monocentric city setup. ${ }^{7}$ However, these papers regard the mechanism of knowledge spillovers as exogenously given, thereby ruling out any two-way interactions between endogenous patterns of knowledge transmission and population agglomeration. This unexplored issue is the main focus of the present paper.

The remainder of the paper is organized as follows. Section II describes the economy's physical environment. In Section III, we consider determination of the economy's steady-state equilibrium in a closed-city (fixed population) model. This allows us to identify the effects of the level of agglomeration on the economy's endogenous patterns of knowledge exchange. In the next section, we simultaneously determine the patterns of interaction and the economy's population size. Section IV also examines the optimality properties of the decentralized equilibrium obtained in Section III. Section V concludes. The Appendix contains some extensions and additional mathematical details.

\section{The Basic Structure of the Economy}

This section specifies the economic environment and outlines the mechanisms through which knowledge spillovers occur among agents. We use a continuous-time framework where each infinitelylived agent has an identical discount rate of $r>0$.

\section{II.A. Economic Agents}

Our goal is to investigate the impact of heterogeneity on the patterns of knowledge accumulation, as well as its interactions with agglomerative activity. To this end, we emphasize the 'horizontal' aspects of knowledge rather than its 'vertical' aspects. ${ }^{8}$ Each agent is endowed with a specific type of knowledge

${ }^{7}$ Similar knowledge spillover factors are also considered in Black and Henderson $(1998,1999)$ in an urban economics context.

${ }^{8}$ Hence, our approach differs from the work of Jovanovic and Rob (1988) in which agents exhibit heterogeneity only in their "vertical" aspect of knowledge (i.e., same type but different quality). 


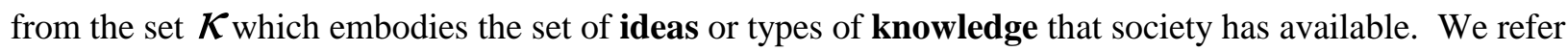

to $\mathcal{K}$ as the "knowledge space" of the economy. The knowledge space may contain any fields of relevance, such as art, biology, history, physics, and economics. As agents in this economy might be regarded as individual workers/consumers, firms, or patent holders, one could interpret $k \in \mathcal{K}$ as an individual's primary field of expertise.

We make the following additional assumptions about the economy. First, there is a continuum of agents in the economy with a total population of Lesbesgue measure $N$. Second, we assume that agents' knowledge types are uniformly distributed across the economy's knowledge space. In addition, the knowledge space, $\boldsymbol{K}$, is a circle of unit circumference. Please refer to Figure 1 where the knowledge space is depicted. Each point along the circle indicates a particular knowledge type. For illustrative purposes, Figure 1 highlights two specific knowledge types $k$ and $k$ '. As we describe above, this could represent two different fields such as art and biology. Finally, note that since $N$ is the total population in the economy and knowledge types are uniformly distributed across $\mathcal{K}$, the density of individuals of each knowledge type is also given by $N$.

\section{II.B. Intellectual Exchange}

Agents can meet with others, collaborate and share their ideas to create new knowledge, which enables them to produce more effectively when matched. To begin, consider two individuals $k$ and $k^{\prime} \in \mathcal{K}$ currently matched and exchanging information with each other. Obviously, heterogeneity among agents plays an important role in sharing information and generating new knowledge. To model the effects of heterogeneity on the knowledge exchange process, we consider the following possibility. When individuals are too alike, they cannot accomplish much and little new knowledge will be created. In 
contrast, if individuals are too different, they will not have productive exchange. This latter point can be envisioned by contemplating the results of a match between a brain surgeon and an opera singer, as they have little in common to communicate and hence nothing to exchange. ${ }^{9}$

Therefore, it is important to define a distance measure in the knowledge space. Let the knowledge distance between $k$ and $k^{\prime} \in \mathcal{K}$ be measured by the Euclidean metric $d\left(k, k^{\prime}\right) .{ }^{10}$ Under our construction regarding the efficacy of knowledge exchange, it is natural to assert there is an optimal level of ideadiversity among agents denoted by $\bar{d}$. Specifically, knowledge creation is increasing in $d$ for $d<\bar{d}$ but decreasing in $d$ for $d>\bar{d}$.

The additional knowledge obtained by an individual $k$, when collaborating with another individual $k^{\prime}$, is denoted as $S\left(k, k^{\prime}\right)$ and is given by:

$$
S\left(k, k^{\prime}\right)=q_{0}+s_{0}\left(a_{0}-a_{1}\left|\bar{d}-d\left(k, k^{\prime}\right)\right|\right)
$$

The term $q_{0}$ refers to the additional knowledge that an agent obtains from a match independent of the knowledge type of a partner. The parameter $a_{l}$ reflects the sensitivity of knowledge creation to heterogeneity among agents with different types of expertise or ideas. Finally, $a_{0}$ reflects the maximum knowledge creation that results from differences in ideas while $s_{0}$ is a positive scaling factor for knowledge exchange. We assume throughout that each parameter in $S\left(k, k^{\jmath}\right)$ is non-negative.

For clarity, we illustrate the role of heterogeneity among agents for knowledge creation in Figure 2. This figure is depicted from the perspective of an individual of knowledge type $k$. The horizontal axis of Figure 2 represents the set of knowledge types that the individual may meet. The vertical axis gives the

${ }^{9} \mathrm{We}$ show in an extension in the Appendix that our results are robust to the alternative possibility that knowledge creation is montonically more effective when agents are alike.

${ }^{10}$ In general, one may define an individual's knowledge expertise as a set. Such generalization would, however, require the adoption of Hausdorff metric to measure the knowledge distance between different sets of individual knowledge. For simplicity, the present paper therefore labels agents by a single point representing their expertise, allowing us to adopt the conventional Euclidean metric to measure the distance between two individuals in knowledge space. 
flow value of matching with each type of agent. Figure 2 emphasizes that agents would generate the most new knowledge upon producing with an individual who is $\bar{d}$ units away in idea space. For an individual of knowledge type $k$, Figure 2 shows that the best matches would occur upon meeting with either an individual of knowledge type $k-\bar{d}$ or $k+\bar{d}$.

We briefly summarize the important concepts regarding intellectual exchange. In our model, heterogeneity in terms of agents' types of knowledge affects the efficacy of exchanging information and producing with others. As one possibility for understanding the role of heterogeneity for knowledge exchange, we initially assume that knowledge exchange is less effective when meeting with individuals who are too alike or too different. To formalize this idea, we postulate that there is an optimal level of idea-diversity among agents which we denote as $\bar{d}$. This assumption is just a simple way of attempting to uncover the impact of heterogeneity among agents on the process of knowledge creation and human capital accumulation in actual economies. ${ }^{11}$ Finally, since the additional knowledge obtained through matching depends on the distance between $d\left(k, k^{\prime}\right)$ and $\bar{d}$, we find it useful to refer to the distance, $\left|\bar{d}-d\left(k, k^{\prime}\right)\right|$, as an agent's "knowledge spread". We denote the knowledge spread as $\delta(k, k$ ').

\section{II.C. Production and Tastes}

By meeting and exchanging ideas with each other, individuals enhance their ability to produce a homogeneous consumption good. With their additional knowledge stock, $S\left(k, k^{\prime}\right)$, agents produce flow output, $y\left(k, k^{\prime}\right)$ given by:

$$
y\left(k, k^{\prime}\right)=A S\left(k, k^{\prime}\right)
$$

${ }^{11}$ Admittedly, our structure has two limitations in order to provide tractability. On the one hand, we do not allow for an individual-specific quality measure which may play a role in affecting the efficacy of knowledge creation (e.g., a high ability agent may gain little from a low ability agent regardless of their knowledge heterogeneity). On the other, new knowledge obtained from matching does not permanently augment an individual's human capital level. Modeling the dynamic evolution of knowledge would lead to a distribution of human capital levels of agents due to random matching. The latter consideration, in particular, would render the model less tractable without adding additional insights into understanding agglomerative activity. 
where $\mathrm{A}>0$ is a scaling factor capturing the overall level of technology in the economy. In addition, everyone in the economy has the same preferences over the homogeneous consumption good with flow utility given by:

$$
u(y)=y
$$

where $y$ is the consumption of output which occurs upon matching and creating new knowledge. There is no disutility of effort. Moreover, flow utility is intertemporally separable. Individuals make choices, as described below, to maximize their expected lifetime utility.

\section{II.D. Matching}

In our economy, agents are randomly matched. An agent searches for partners to meet and exchange ideas. In order to illustrate how a dense economic environment fosters more opportunities for interaction, we assume that the flow probability for an unmatched agent to locate or meet another unmatched agent is given by $\alpha(U)$, with $\alpha_{U} \geq 0$, where $U$ is the mass of unmatched individuals in the economy. ${ }^{12}$ With a larger mass of individuals residing in a given physical area, individuals interact more frequently which results in a higher arrival rate of potential partners for knowledge exchange. The idea that population density may stimulate knowledge creation is emphasized in Marshall (1890), Kuznets (1962), Pred (1966), and Jacobs (1969). One could also adopt an alternative interpretation of $\alpha(U)$ as the amount of time it takes for information flows to accrue across sectors in an economy rather than an explicit search-theoretic interpretation. ${ }^{13}$

Further, given the effects of heterogeneity on the efficacy of knowledge exchange in this economy, it is important to distinguish between meetings and matches. Meetings occur between any two agents with

\footnotetext{
${ }^{12}$ This assumption follows the specification of the arrival rate in Diamond (1982). It implies that the arrival rate of meetings is higher in economies with a higher population density.

${ }^{13}$ This is, in fact, the interpretation adopted by Alfred Marshall, “...so great are the advantages which people following the same skilled trade get from near neighbourhood to one another. The mysteries of the trade become no mysteries; but are as it were in the air, and children learn many of them unconsciously." (1895, p.352)
} 
flow probability $\alpha(U)$, but only a subset of meetings result in matches. This is because agents do not want to produce with individuals whose areas of expertise are too alike or too different, which would result in less effective knowledge exchange. We therefore denote the endogenous flow probability of a match for an individual agent $k$ as $\beta\left(\delta_{k} ; U\right)$. Of course, as we demonstrate below, $\beta\left(\delta_{k} ; U\right)<\alpha(U)$.

First, note that $\beta\left(\delta_{k} ; U\right)$ will depend on the range of types of knowledge that an agent $k$ accepts for intellectual exchange. Specifically, $k$ will select a range of agents with whom to exchange ideas given by: $R(k) \equiv\left[k-\bar{d}-\delta_{k}, k-\max \left\{0, \bar{d}-\boldsymbol{\delta}_{k}\right\}\right] \cup\left[k+\max \left\{0, \bar{d}-\boldsymbol{\delta}_{k}\right\}, k+\bar{d}+\boldsymbol{\delta}_{k}\right]$, as depicted in Figure 3. Thus, in computing the flow probability of a match, $\beta\left(\delta_{k} ; U\right)$, we may write:

$$
\beta\left(\delta_{k} ; U\right)=\alpha(U)\left(\frac{\int_{R(k)} U d k^{\prime}}{U}\right)
$$

Note that the term $\int_{R(k)} U d k^{\prime}$ reflects the total mass of individuals that agent $k$ selects to try to match. This is divided by the total mass of unmatched agents to obtain the proportion of unmatched agents in the economy that agent $k$ selects to try to engage in intellectual exchange. The flow probability of a match is thus given by the flow probability of a meeting multiplied by the proportion of unmatched agents selected for knowledge exchange. In addition, matches between agents are terminated with an exogenous flow probability $\eta$, i.e., the exogenous detachment rate in the economy is given by $\eta .{ }^{14}$ If two individuals meet, but do not match, detachment occurs immediately.

The selection strategy determining which agents are accepted for matches will depend on both the effectiveness of knowledge exchange and primitives of the economic environment such as the ability of individuals to meet in the economy. For example, as it becomes easier for unmatched agents to meet,

\footnotetext{
${ }^{14} \mathrm{An}$ exogenous separation rate provides tractability. We could endogenize the separation rate along the lines of Jovanovic (1979) if the productivity of each match is not known ex-ante and agents update their beliefs regarding the productivity of a match over time. Once an agent determines that a match is not sufficiently worthwhile, endogenous separation would occur. This extension is not likely to add additional insights into the fundamental issues we study.
} 
individuals would be expected to be more selective in the range of agents they will accept for engaging in knowledge exchange.

\section{II.E. Asset Values}

Recall that in any time period, agents will either be matched or unmatched. Each state is associated with different levels of expected lifetime utility because agents' consumption opportunities will vary depending on whether they are currently matched or not. Therefore, let $V_{M t}\left(k, k^{\prime} ; U\right)$ denote the expected lifetime utility for an agent of knowledge type $k$ who is currently matched with an agent of knowledge type $k^{\prime}$ in time period $t$. The expected lifetime utility for an unmatched agent of knowledge type $k$ is given by $V_{U t}(k ; U)$ in time period $t$.

We begin by describing the evolution of the expected lifetime utility for an agent of knowledge type $k$. A derivation of the agent's Bellman equation is easiest to see by considering time in discrete units of length $\Delta .{ }^{15}$ Under this time convention, the expected lifetime utility for an agent who is currently matched in time period $t$ is given by:

$$
V_{M t}=\frac{\eta \Delta\left[y\left(k, k^{\prime}\right) \Delta+V_{U(t+\Delta)}\right]+(1-\eta \Delta)\left[y\left(k, k^{\prime}\right) \Delta+V_{M(t+\Delta)}\right]}{1+r \Delta}
$$

where under the Poisson assumption for the separation rate, the probability that a breakup will occur within the time interval $\Delta$ is given by $\eta \Delta$. Until the break-up occurs, it is assumed that agents will be exchanging information and producing. As of period $(t+\Delta)$, the individual will have an expected lifetime utility of $V_{U t+\Delta}(k ; U)$. In contrast, with probability $(1-\eta \Delta)$, agents will remain matched and therefore have an expected discounted lifetime utility of $V_{M t+\Delta}\left(k, k^{\prime} ; U\right)$ as of period $(t+\Delta)$. Rearranging the above expression for $V_{M t}\left(k, k^{\prime} ; U\right)$, dividing by $\Delta$, and taking limits as $\Delta \rightarrow 0$ yields:

$$
r V_{M t}=y\left(k, k^{\prime}\right)+\eta\left[V_{U}-V_{M}\right]+\dot{V}_{M t}
$$

\footnotetext{
${ }^{15}$ See Trejos and Wright (1995) for an additional example in a search-theoretic model of money.
} 
In a steady-state, the expected lifetime utility of an agent is independent of time. Thus, the Bellman equation for an agent of knowledge type $k$ who is currently matched with an agent of type $k$ ' is:

$$
r V_{M}\left(k, k^{\prime} ; U\right)=y\left(k, k^{\prime}\right)+\eta\left(V_{U}(k ; U)-V_{M}\left(k, k^{\prime} ; U\right)\right)
$$

This implies that the flow value of matches is the sum of the flow output produced based on new knowledge created and the expected capital loss associated with the change of state from a matched to an unmatched agent.

Analogously, we can express the corresponding Bellman equation for unmatched agents of type $k$. It is derived in the same manner as the Bellman equation for matched agents. Given the set of knowledge types, $R(k) \equiv\left[k-\bar{d}-\delta_{k}, k-\max \left\{0, \bar{d}-\delta_{k}\right\}\right] \cup\left[k+\max \left\{0, \bar{d}-\delta_{k}\right\}, k+\bar{d}+\boldsymbol{\delta}_{k}\right]$ (see Figure 3 ), that an individual of knowledge type $k$ selects for matching, the Bellman equation for an unmatched agent of type $k$ is:

$$
r V_{U}(k ; U)=\alpha(U) \int_{R(k)}\left(V_{m}\left(k, k^{\prime} ; U\right)-V_{U}(k ; U)\right) d k^{\prime}
$$

It is important to note that the selection strategy will be chosen by recognizing the tradeoff between a higher number of matches (a contact rate effect) and more effective matches (a knowledge efficacy effect) because knowledge creation would not be effective when producing with agents who are too similar or too different.

\section{II.F. Steady-State Populations}

In any time period, agents will either be matched or unmatched. Without imposing additional $a$ priori heterogeneity in the knowledge space, we assume that the distribution of unmatched (and thus matched) agents is uniform. Let $M$ be the mass of matched individuals, where $M=N-U$. Constant population in the steady state therefore requires that the inflows and outflows of population category are equal in each time period.

For each type of agent, there is a given mass of unmatched individuals $U_{k}$. From this pool of unmatched agents, the flow probability that an agent will find a suitable match is given by $\beta\left(\delta_{k} ; U\right)$. Suppressing arguments for notational convenience, this implies that an outflow of $\beta\left(\delta_{k} ; U\right) U_{k}$ agents of 
type $k$ from the unmatched pool will become matched within the period. Similarly, given the exogenous detachment rate $\eta$, an inflow of $\eta M_{k}$ agents of type $k$ will enter the unmatched pool. Since we intend to examine a symmetric, steady-state equilibrium we require the following in order for the populations of matched and unmatched agents to remain constant over time:

$$
\beta(\delta ; U) U=\eta M=\eta(N-U) .
$$

From (7) we find that $U=\frac{\eta}{\beta(\delta, U)+\eta} N$. Individuals regard their own selection of the knowledge spread as having no influence on the steady-state population of unmatched agents.

\section{Steady-State Equilibrium}

In this section, we focus on determining the steady-state equilibrium in the context of an environment where the population mass is exogenously given. This allows us to highlight the influence of the extent of agglomeration on the knowledge creation process, as well as to obtain insights concerning the knowledge spread. Throughout, we assume that:

Assumption 1. (Knowledge Diversity) The optimal level of idea-diversity satisfies ${ }^{16}$ :

$$
\bar{d} \geq \frac{2\left(q_{0}+s_{0} a_{0}\right)}{s_{0} a_{1}}
$$

Consider,

Definition 1: (Steady-State Equilibrium) A non-degenerate, symmetric, steady-state equilibrium (SSE) is a tuple $\left\{\{R(k)\}_{k \in \kappa}, \hat{\delta}, U\right\}$ satisfying the following conditions:

(E-1) agents maximize their expected lifetime utilities through their choice of the knowledge spread,

${ }^{16}$ From the definition of $R(k)$, it is clear that the agent's unmatched value function will have a kink at $\delta_{k}=\bar{d}$. Under Assumption 1, however, this kink will occur where $V_{U}\left(\delta_{k} ; U\right)$ is negative. Therefore, the value of $\bar{d}$ does not affect the agent's choice of her knowledge spread. This considerably simplifies the analysis. The Appendix presents some additional technical details for the case where Assumption 1 does not necessarily hold. 
that is $\hat{\delta}_{k}$ is such that: $\hat{\delta}_{k} \underset{\delta_{k}}{\operatorname{argmax}} V_{U}\left(\delta_{\mathrm{k}} ; U\right)$, where the value function $V_{U}$ satisfies (5) and (6);

(E-2) equilibrium range of agents for $k$ to exchange ideas:

$$
R(k) \equiv\left[k-\bar{\delta}-\hat{\delta}_{k}, k-\bar{\delta}+\hat{\delta}_{k}\right] \cup\left[k+\bar{\delta}-\hat{\delta}_{k}, k+\bar{\delta}+\hat{\delta}_{k}\right]
$$

(E-3) steady-state population: (7);

(E-4) symmetry: $\hat{\delta}_{k}=\hat{\delta}, \forall k \in \boldsymbol{K}$;

(E-5) there is interaction among agents (the steady-state equilibrium is non-degenerate): $\hat{\delta}>0$

Notice that there is an ex-ante optimal level of idea heterogeneity, $\bar{d}$, between any two matched parties. Based on parameters of the model such as matching rates, agents establish the maximal distance $\hat{\delta}$ away from $\bar{d}$ to match. As illustrated in Figure 1, this determines the equilibrium range of agents with whom agent $k$ exchanges ideas. Upon deriving the range of individuals for whom agents match, we can pin down the degree of diversity of knowledge exchange in this economy in steady-state equilibrium. Once the equilibrium knowledge spread, $\hat{\delta}$, is determined, the steady-state populations of matched and unmatched agents can be derived using (7). Symmetry is also important here. Any time when two agents meet, they both have the same possible gains from matching. Therefore, if an agent of type $k$ wants to match with an agent of type $k^{\prime}$, then type $k^{\prime}$ also wants to match with type $k$. We can then establish:

Theorem 1: (Existence) Suppose that Assumption 1 holds and $s_{0}$ and $a_{1}$ are strictly positive. Under these conditions, a (non-degenerate), symmetric, steady-state equilibrium exists and is unique.

Proof. All proofs are in the Appendix.

Upon establishing existence of the steady-state equilibrium for the economy, we seek to understand how the pattern of information flows, as exhibited by the knowledge spread, responds to the extent of agglomeration, as measured by the exogenous population size in the basic model. We can show: 
Proposition 1: (Effect of the extent of agglomeration on knowledge exchange pattern) Economies with a higher population mass have a smaller equilibrium knowledge spread.

Intuitively, this occurs because the probability of finding other unmatched agents is higher in economies with a higher population mass. As a consequence, agents are more selective in knowledge exchange. The main implication of this result is that with population agglomeration, knowledge spillovers are likely to be more specialized. Interestingly, these benefits associated with specialization are different than those suggested by Adam Smith. Although Smith posited that internal economies of scale may drive specialization and the division of labor, we show that specialization can also be driven by external effects due to matching. A higher density of workers implies that for any given matching range, the likelihood of finding an acceptable partner will be higher.

It may be noted that as knowledge itself becomes more specialized, knowledge exchange with agents in other fields become less effective. This is captured in our model by a higher penalty for heterogeneity $\left(a_{1}\right)$, which induces agents to become more selective in matching. However, the overall level of technology (A) has no effect on the equilibrium knowledge spread. A higher level of technology raises the productivity of each match, but it also raises the costs of a higher knowledge spread because agents forfeit production that would have been obtained from more effective knowledge exchange with other agents. The remaining details concerning comparative statics in the closed-city model can be found in the Appendix.

\section{Endogenous Migration}

In this section, we discuss equilibrium determination of the population size along with the steadystate equilibrium knowledge spread. In contrast with the benchmark case investigated in Section III, where the population mass is exogenously given, we pin down the equilibrium knowledge spread along with the endogenous population mass. This framework provides numerous insights into the interactions between 
the patterns of knowledge exchange and the process of agglomeration. We demonstrate that these considerations identify new sources of inefficiency associated with population migration. In particular, individuals fail to take into account the potential effect of the selection of their knowledge spread on others, which we refer to as the matching externality effect. The matching externality effect provides a channel that suggests the equilibrium population size may fall below its socially optimal value. We also find a congestion externality exists in the economy. Although the existence of congestion externalities is standard in the conventional urban economics literature, we show this effect also has implications for the patterns of knowledge exchange. ${ }^{17}$ In particular, we demonstrate that the congestion effect causes individuals to under-search by over-specializing in knowledge exchange.

In regard to migration decisions, individuals must account for costs associated with residing in the city under consideration. These costs (which are asserted to depend positively on $N$ ) may, for example, be seen as the (present-discounted) value of city taxes imposed on each immigrant. A higher $N$ may be associated with congestion costs or destruction of existing structures, thus requiring government infrastructure spending to increase at least proportionately (i.e., a higher $N$ leads to the same or an increase in real per capita infrastructure costs). ${ }^{18}$ In short, we specify $v=v(N)$ as the (average) entry cost function, where $v$ is strictly increasing (and convex) in $N$. For comparative statics exercises, we view any increase in costs associated with residing in the city as an increase in 'entry' costs and represent them as a shift in the entry cost function.

Please refer to Figure 4 to better understand how the endogenous population mass is determined.

\footnotetext{
${ }^{17}$ See chapter 6 of Fujita (1989) for details of the conventional model that results in cities that are overpopulated in equilibrium relative to the social optimum. There are some models that generate cities that are underpopulated in equilibrium relative to the optimum. In the presence of either a fixed set-up cost [Abdel-Rahman (1990)] or a free-rider effect [Palivos and Wang (1997)], the equilibrium city size may be too small. Within our general equilibrium search-theoretic framework, channels for either over-population or under-population are present.
}

${ }^{18}$ See Chapter 6 of Fujita (1989) for a discussion of congestible city goods. 
The horizontal axis of Figure 4 represents different values of the population mass, $N$, and the vertical axis provides the different values for an unmatched agent's expected lifetime utility unmatched and entry costs for each value of $N$. In our analysis, an agent's unmatched value function is expressed in terms of $N$ by substituting for $U$ from the steady-state population condition (7). Individuals will continue to migrate as

long as $V_{U}(\delta ; N) \geq v(N) .{ }^{19}$ For values of $N$ less than $\tilde{N}, V_{U}(\delta ; N)>v(N)$, providing an incentive for agents to move to the area. If $N$ is greater than $\tilde{N}$, agents would obtain higher expected lifetime utility by choosing not to migrate to the area. Thus when the extent of agglomeration is such that $V_{U}(\delta ; N)=v(\tilde{N})$, migration will no longer occur. For these reasons, we refer to the condition that $V_{U}(\delta ; N)=v(\tilde{N})$ as the equilibrium entry condition. It may also be useful to think of the condition $V_{U}(\delta ; N)=v(\tilde{N})$ as the endogenous population condition. From the equilibrium entry condition, we can obtain a locus of $\delta$ and $N$ where individuals are indifferent between migrating and not migrating. Through this endogenous migration choice, the population mass is pinned down for each possible value of the knowledge spread. In combination with the knowledge spread locus, we are able to obtain a steady-state equilibrium allowing for endogenous migration.

\section{IV.A. Steady-State Equilibrium with Endogenous Migration}

The above elements forge our definition of a steady-state equilibrium with endogenous migration. Consider,

Definition 2: (Steady-State Equilibrium with Endogenous Migration) A non-degenerate, symmetric steady-state equilibrium with endogenous migration (SSEEM) is a SSE $\left\{\{R(k)\}_{k \in \mathrm{K}}, \hat{\delta}, \hat{U}\right\}$ together with a population mass $\hat{N}$ satisfying the following additional conditions:

(E-4) equilibrium entry: $V_{U}\left(\hat{\delta_{k}} ; \hat{U}\right)=v(\hat{N}) \forall k \in \mathcal{K}$;

${ }^{19}$ Our model of endogenous migration is an open city model. We could close the model using a system of cities approach where migration across locations may occur until equilibrium is achieved. 
(E-5) population agglomeration occurs (the steady-state equilibrium is non-degenerate): $\hat{N}>0$.

We illustrate our solution algorithm through the use of Figure 5 where the horizontal axis represents the different values of an agent's knowledge spread and the vertical axis lists values of $N$. We first derive the knowledge spread (KS) locus which determines the choice of the knowledge spread $\delta$ by each agent for a given size of population mass $N$. We next determine the values of $\delta, U$, and $N$ that keep agents indifferent between migrating and not migrating to the area, i.e., equilibrium entry of agents from Figure 4. For tractability, we focus our attention below on the case where the entry cost function is linear in the population size (in other words, per capita entry costs are constant). We refer to the equilibrium entry (EE) locus as the relationship between the total population, the mass of unmatched agents and the knowledge spread such that individuals are indifferent between migrating or not migrating to the city. A steady-state equilibrium with endogenous migration occurs for values of the knowledge spread and population mass where the equilibrium entry and knowledge spread loci intersect.

Theorem 2: (Existence under Endogenous Migration) Suppose that Assumption 1 holds and $s_{0}$ and $a_{1}$ are strictly positive, a (non-degenerate) steady-state equilibrium with endogenous migration exists and is unique.

We continue by outlining some interesting connections between knowledge exchange and endogenous agglomeration. Additional results are presented in the Appendix.

Proposition 2: (Interactions between the pattern of knowledge exchange and the extent of agglomeration) With endogenous migration, any factor that promotes knowledge exchange will also encourage a higher population mass.

A higher arrival rate $(\alpha)$, for example, implies that it takes less time on average for individuals to meet others. As a result, agents can choose to be more selective and concentrate on finding more effective 
opportunities for knowledge exchange. This immediately implies there are higher gains from migrating because there is less delay between meetings, thereby resulting in a higher population mass.

In contrast with the closed-city model in Section IV, we find that more technologically advanced cities will have more specialized patterns of information sharing. This occurs because the higher level of technology has the direct effect of inducing more population agglomeration. Because of the beneficial aspects of population density for matching, agents in turn decide to become more specialized in their matching. Similar results emerge in regard to the effect of different parameter values concerning the effectiveness of knowledge exchange. According to our closed-economy analysis, a higher penalty for heterogeneity $\left(a_{l}\right)$ induces agents to become more selective in matching. This result does not remain robust to allowing for population migration. Due to the negative effects of the penalty for migration incentives, less agents will decide to migrate. With a lower population mass, there is a lower population density, making it difficult to meet other agents. As a result, agents increase their knowledge spread in equilibrium. An Appendix contains a proof and further details of comparative statics for the open city model.

\section{IV.B. Socially Optimal Knowledge Spread and Population Mass}

In Section IV.A., we analyzed the various two-way interactions between the endogenous knowledge transmission mechanism and the process of population agglomeration. In this section, we demonstrate that these interactions lead to new sources of inefficiency associated with population migration.

In a decentralized equilibrium, individuals choose their knowledge spread to maximize their unmatched value function given the population size, and continue to migrate until the net utility from migrating is equal to zero. In a social planner's problem, we assume that the city planner (or the immigration officer) seeks to maximize the net welfare of a representative city resident over (i) the knowledge spread and (ii) the population mass (or mass of unmatched agents) to be established in which 
all residents simultaneously move to the city. ${ }^{20}$ Define:

Definition 3: (Social Optimum) A symmetric social optimum (SO) is a triple $\left\{\delta^{*}, U^{*}, N^{*}\right\}$ satisfying the following conditions:

(S-1) optimal knowledge spread and population size: $\left\{\boldsymbol{\delta}^{*}, N^{*}\right\} \in\left\{\underset{\delta, N}{\operatorname{argmax}}\left[V_{U}(\boldsymbol{\delta} ; U)-v(N)\right]\right\}$ where $V_{U}$ is defined as in (8);

(S-2) steady-state population: (7).

Theorem 3: (Existence of a Social Optimum) Suppose that Assumption 1 holds and that $s_{0}$ and $a_{1}$ are strictly positive. Under these conditions, a social optimum exists and is unique.

Thus, the social planner chooses $\delta$ and $N$ simultaneously to maximize the net welfare of a representative resident. Because the arrival rate depends on the mass of unmatched agents, it is more convenient to transform the problem such that the social planner chooses $\delta$ and $U$ to maximize the net utility of a potential representative resident. Then, by applying the steady-state population condition, (7), we can solve for $N .^{21}$ As we demonstrate below, the situation where $\alpha$ is a function of $U$ implies a matching externality occurs in equilibrium. This, in turn, distorts the economy's extent of population agglomeration.

${ }^{20} \mathrm{We}$ make two clarifying remarks. First, we use our definition of a social optimum instead of a Pareto optimum as our welfare criterion. In particular, the latter concept need not be well-defined in an "open city" model with migration, since the set of agents present in the model is ill-defined. Second, we consider the case where a city planner chooses to maximize the net welfare of only the individuals residing in the city; the city does not yet exist when the planner solves the optimization problem. One may also consider the case in which the city does exist and the social planner maximizes the welfare of current residents and potential immigrants. This entails consideration of redistribution issues which detract from our principal interest--the interactions between the social inefficiencies from knowledge exchange and congestion externalities.

${ }^{21}$ Mathematically, this is isomorphic to solving for $\delta$ and $N$ first, which provides a solution for $U$ in a recursive manner, though the transformed problem is more tractable. 
We can compare the decentralized equilibrium with the social optimum to conclude:

Proposition 3: (Social inefficiency) Compared to the social optimum, a decentralized equilibrium SSEEM may be under-populated and under-specialized in knowledge exchange or over-populated and over-specialized.

We shall illustrate this result graphically after some discussion. In our model, social inefficiency arises for two reasons. First, the city may feature an equilibrium population mass larger than the social optimum because individuals do not consider their impact on the reservation utility level. By not taking into account their effect on the overall population mass, the city may be over-populated relative to the social optimum. This result occurs in much of the conventional urban economics literature--we show, however, this congestion externality leads to an additional distortion in the pattern of information flows in an economy. In particular, we demonstrate that the congestion externality may cause individuals to under-search for potential collaborators by over-specializing in knowledge exchange.

Second, there is another possible inefficiency when the gains from a higher population density are sufficiently large. The selection of the knowledge spread induces a matching externality in the economy. This occurs because agents fail to account for the fact that accepting matches with more types of individuals lowers the mass of unmatched agents in the economy, rendering it more difficult for everyone to meet other unmatched agents. This potentially results in a decentralized equilibrium that is underspecialized in its patterns of knowledge exchange and under-populated relative to the social optimum. ${ }^{22}$

More specifically, the first-order condition for the social planner's choice of the knowledge spread is:

$$
\left.\frac{\partial V_{U}(\delta, \theta)}{\partial \delta}\right|_{\bar{U}}+\frac{\partial V_{U}(\delta, \theta)}{\partial U} \frac{\partial U}{\partial \delta}=0
$$

\footnotetext{
${ }^{22}$ Burdett and Coles (1997) demonstrate the existence of a sorting externality in their analysis of marriage markets.
} 
Note that the first term in (9) corresponds to the choice of the individual's knowledge spread in a decentralized equilibrium. Individuals, taking the mass of unmatched agents as given, choose the knowledge spread to maximize their expected lifetime utility. The social planner, however, takes into account that a larger knowledge spread lowers the mass of unmatched agents in the economy (which we refer to as the matching externality effect). This is the second term in equation (9).

As stated in Proposition 3, a decentralized equilibrium may be under-populated and underspecialized in knowledge exchange or over-populated and over-specialized relative to the optimum. We can illustrate both cases by extending our analysis in Figure 3 using Figures 6 and 7. To introduce these arguments graphically, we need to introduce some additional notation. Denote $V_{U}(\hat{\delta} ; N)$ as an unmatched agent's expected lifetime utility given the private choice of the knowledge spread. In addition, let $V_{S P}\left(\delta^{*}\right.$, $N$ ) denote an unmatched agent's expected lifetime utility under the planner's choice of the knowledge spread, $\delta^{*}$. Next, please refer to Figure 6 where the horizontal axis gives the population mass, $N$, and the vertical axis gives the entry cost $(v)$, and expected lifetime utilities for unmatched agents under the private and planner's choice of the knowledge spread $\left(V_{U}\right.$ and $\left.V_{S P}\right)$.

We first consider the case where the matching externality is minimal so that the planner's choice of the knowledge spread is not too much smaller than in equilibrium. Since the matching externality in this case is not too strong, an agent's unmatched value function $\left(V_{U}\right)$ will not lie much below the lifetime utility that would occur (for each value of $N$ ) under the planner's choice of the knowledge spread $\left(V_{S P}\right)$. Recall that under endogenous migration, the steady-state equilibrium population level is pinned down where the unmatched value function intersects with the entry cost function, $\hat{N}$. For the social optimum, however, the population level is found where the slope of the unmatched value function with respect to $N$ has the same slope as the entry cost function, $N^{*}$. In this case, we obtain the standard over-population result. Now refer to Figure 7. In contrast, when the matching externality effect is strong, the equilibrium 
value of the knowledge spread is small relative to the optimum. As a consequence, $V_{S P}$ may lie far above $V_{U}$ and the optimal population $N^{*}$ exceeds the equilibrium population $\hat{N}$.

\section{Concluding Remarks}

This paper develops a search-theoretic model in order to examine the patterns of knowledge exchange and its interaction with agglomerative activity. In particular, we illustrate heterogeneity (in terms of agents' types of knowledge) plays a role in the acquisition and the advance of knowledge. This occurs because agents in our model face a trade-off between specialized, highly beneficial knowledge exchange and a higher number of matches.

We initially consider an environment in which the population size is fixed and examine the role of agglomeration for an economy's endogenous pattern of knowledge exchange. We demonstrate that economies with higher search or market frictions will have more diversified patterns of information exchange. Individuals favor obtaining a higher quantity, but less effective interactions with others in economies with higher search frictions. In this way, we examine the role of market frictions for human capital accumulation and an economic growth. We also find that cities with a higher population size will have more specialized patterns of information flows. This leads to a potentially testable hypothesis of the theory, namely that the degree of specialization in a city and its population are positively correlated. We shall return to this momentarily.

We later examined the interaction between the patterns of knowledge exchange and the endogenous spatial agglomeration of an economy. This analysis demonstrates how information exchange affects population agglomeration. We show technologically advanced economies will have a higher population size and more specialized patterns of information exchange.

In extensions outlined in the Appendix, we further examine the role of geography for the advance of knowledge and agglomeration. In this manner, we also establish microfoundations for human capital spillovers which may take place across different locations as in the work of Eaton and Eckstein (1997). In 
addition, we demonstrate that economies with higher transportation costs feature less interactive activity. Since the existing urban economics literature ignores this effect, it is likely that the social costs of transportation or commuting are under-stated. In a second extension, we describe how our basic insights are robust to the alternative possibility that knowledge exchange is monotonically more effective when agents are alike $(\bar{d}=0)$.

In a third extension found in the Appendix, we link an economy's overall measure of diversity to its patterns of knowledge exchange. Due to self-fulfilling prophecies, we find that multiple equilibria exist in which economies with the same population size may be either specialized or diverse. We show that specialized cities will have specialized patterns of interaction while agents in diverse cities settle for more matches at the expense of less beneficial knowledge exchange. Because these equilibria are Paretorankable, we obtain a role for city planners to induce the economy to select a more specialized set of industries and thereby encourage more effective means of learning from others.

We believe there are a number of interesting issues which may be pursued in this research. The first objective is to explore the interactions between knowledge exchange and agglomerative activity in environments where individuals have different levels of human capital. This would allow for a rich array of possible interactions among agents due to 'horizontal' and 'vertical' aspects of knowledge and agglomerative activity. Second, one may seek to investigate the relationships between knowledge exchange and agglomerative activity when individuals make human capital investments prior to engaging in the exchange of information. In this manner, the benefits of agglomeration due to lower costs of communication in dense environments will affect initial human capital decisions.

An important objective of our research is examining the implications of horizontal differences in knowledge for patterns of information exchange and agglomerative activity. In particular, our model demonstrates that larger cities should have more specialized patterns of information flows due to lower costs of communication in dense economic environments. With these insights, we believe it would be 
interesting to reconsider the evidence on knowledge spillovers using patent data as in Jaffe et al (1993, 1996). This might begin by examining if larger cities have more specialized knowledge exchange.

These directions for research will provide additional insights into the connections between local economic growth and agglomeration. 


\section{Appendix A}

1. Derivation of Agents' Unmatched Value Function in Section II.

Manipulating the Bellman equation for matched individuals, equation (4) yields:

$$
V_{m}\left[\left(k, k^{\prime}\right) ; U\right]=\frac{y\left(k, k^{\prime}\right)+\eta V_{U}(k ; U)}{r+\eta}
$$

Recall that agent $k$ will agree to match and exchange ideas with any individual $k$ ' belonging to the set $R(k) \equiv\left[k-\bar{d}-\boldsymbol{\delta}_{k}, k-\max \left\{0, \bar{d}-\boldsymbol{\delta}_{k}\right\}\right] \cup\left[k+\max \left\{0, \bar{d}-\boldsymbol{\delta}_{k}\right\}, k+\bar{d}+\boldsymbol{\delta}_{k}\right]$ (see Figure 3). Thus, the Bellman equation for an unmatched agent of type $k$ is:

$$
r V_{U}(k ; U)=\alpha(U) \int_{R(k)}\left(V_{m}\left(k, k^{\prime} ; U\right)-V_{U}(k ; U)\right) d k^{\prime}
$$

Note, however, from the definition of $R(k)$ that an agent's unmatched value function will have a kink where $\delta_{k}=\bar{d}$. We begin by finding $V_{U}\left(\delta_{k} ; U\right)$ over the region where $\delta_{k}<\bar{d}$. Integration from zero to $\delta_{k}$ yields:

$$
V_{U}(\delta ; U)=\left(\frac{4 \alpha(U) \delta_{k}}{r+\eta+4 \alpha(U) \delta_{k}}\right) \frac{A}{r}\left(q_{0}+s_{0} a_{0}-\frac{1}{2} s_{0} a_{1} \delta_{k}\right) \text {, where } \delta_{k}<\bar{d}
$$

Next, denote $\delta_{\max } \equiv \frac{2\left(q_{0}+s_{0} a_{0}\right)}{s_{0} a_{1}}$ which implies that for any value of $\delta_{\mathrm{k}}$ greater than $\delta_{\max }, V_{U}\left(\delta_{k} ; U\right)<0$.

Thus, under Assumption 1, the kink in the value function occurs over a region where an agent's unmatched value is negative and is not important for determining the individual's knowledge spread. For additional details, see the extensions in Appendix C. 


\section{Derivation of the Equilibrium Knowledge Spread and Proof of Theorem 1.}

Differentiating $V_{U}(\delta ; U)$ with respect to $\delta$ provides the first-order condition for obtaining the equilibrium

knowledge spread. Simple algebra yields the following quadratic equation which can be used to derive $\hat{\delta}$ :

$$
\delta^{2}+\frac{b(r+\eta)}{2 \alpha U} \delta-\frac{b(r+\eta)}{2 \alpha} \frac{q_{0}+s_{0} a_{0}}{s_{0} a_{1} U}=0
$$

One can easily solve for the mass of unmatched agents from the knowledge spread to obtain:

$$
U^{K S}=\frac{r+\eta}{\alpha}\left(\frac{\left(q_{0}+s_{0} a_{0}-s_{0} a_{1} \delta\right)}{2 s_{0} a_{1} \delta^{2}}\right)
$$

From the steady-state population condition (7), we obtain:

$$
N^{K S}=\frac{r+\eta}{\alpha \eta \delta}\left(\frac{\left(q_{0}+s_{0} a_{0}-s_{0} a_{1} \delta\right)}{s_{0} a_{1} \delta}\right)^{2}+\frac{r+\eta}{2 \alpha}\left(\frac{\left(q_{0}+s_{0} a_{0}-s_{0} a_{1} \delta\right)}{s_{0} a_{1} \delta^{2}}\right)
$$

It is easy to see the knowledge spread locus is a downward-sloping curve as depicted in Figure 4.

\section{Derivation of the Equilibrium Entry Locus.}

Recall the equilibrium entry locus is a curve of values of the knowledge spread and the population mass where $V_{U}(\delta ; U)=v(N)$. This implies:

$$
V_{U}(\delta ; U)=\left(\frac{4 \alpha(U) \delta}{r+\eta+4 \alpha(U) \delta}\right) \frac{A}{r}\left(q_{0}+s_{0} a_{0}-\frac{1}{2} s_{0} a_{1} \delta\right)=\mathrm{v}_{0} N
$$

Substituting the steady-state population condition (7) for the mass of unmatched agents in (A7) above generates the equilibrium entry locus.

\section{Proof of Theorem 2.}

Recall the first-order condition for the knowledge spread and rewrite it in terms of $V_{U}(\delta ; U)$. Substitute the equilibrium entry condition as in (A7) and the steady-state population condition to obtain:

$$
v_{0}(\eta+4 \alpha \delta U)(r+\eta)=\eta\left(\frac{1}{2} s_{0} a_{1} \delta\right)(4 \alpha \delta)\left(\frac{A}{r}\right)
$$


This yields a relationship between $\delta$ and $U$. We next attempt to substitute this information into the equilibrium entry condition of (A7) to obtain a second equation in terms of $\delta$ and $U$. Substituting (A8) into (A7) generates:

$$
v_{0}(\eta+4 \alpha U \delta)=\frac{(4 \alpha \delta) \eta\left(\frac{A}{r}\right)\left(q_{0}+s_{0} a_{0}-s_{0} a_{1} \delta\right)}{r+\eta+4 \alpha U \delta}
$$

Manipulating (A8) and (A9) yields the following relationship for the steady-state equilibrium knowledge spread under endogenous migration:

$$
\Lambda(\delta) \equiv\left(\frac{A}{r}\right) \eta(2 \alpha)\left(s_{0} a_{1}\right)^{2} \delta^{3}+(r+\eta) s_{0} a_{1} v_{0}(2 r+\eta) \delta-2\left(q_{0}+s_{0} a_{0}\right)(r+\eta)^{2} v_{0}=0
$$

We prove the existence of the steady-state equilibrium knowledge spread using the following mean value argument. First, note that $\Lambda(\delta)$ is continuous in $\delta$. Also, $\Lambda(0)<0$. Since individuals will never choose a knowledge spread which yields zero utility, an upper bound for $\delta$ is given by $\delta_{\max } \equiv \frac{2\left(q_{0}+s_{0} a_{0}\right)}{s_{0} a_{1}}$. Ignoring the cubic term in $\Lambda(\delta)$ reveals that $\Lambda\left(\delta_{\max }\right)$ is positive as long as the discount rate is positive. Therefore, there is a value of $\delta$ where $\Lambda(\delta)=0$ which demonstrates that a steady-state equilibrium under endogenous migration exists. Finally, since $\Lambda(\delta)$ is strictly increasing in $\delta$, there can only be one value of $\delta$ where a steady-state equilibrium under endogenous migration exists.

\section{Proof of Theorem 3.}

The proof of Theorem 3 is similar to the proof of Theorem 2. 


\section{Appendix B}

\section{Additional Comparative Statics Results from Section III.}

The comparative statics of the steady-state equilibrium knowledge spread are as follows:

$$
\frac{\partial \hat{\delta}}{\partial N}<0, \frac{\partial \hat{\delta}}{\partial \alpha}<0, \frac{\partial \hat{\delta}}{\partial \eta}>0, \frac{\partial \hat{\delta}}{\partial A}=0, \frac{\partial \hat{\delta}}{\partial r}>0, \frac{\partial \hat{\delta}}{\partial s_{0}}<0, \frac{\partial \hat{\delta}}{\partial a_{1}}<0, \frac{\partial \hat{\delta}}{\partial q_{0}}>0 .
$$

The results are easy to interpret. As it becomes easier for unmatched agents to find potential partners for knowledge exchange ( $\alpha$ higher), they can concentrate on finding more productive matches. The exogenously given detachment rate, $\eta$, is positively associated with more diverse patterns of knowledge exchange (i.e, a larger knowledge spread). This occurs because higher values of $\eta$ imply that matches do not last as long on average, resulting in a smaller opportunity cost to taking part in relatively less effective knowledge exchange. When the discount rate $(r)$ rises, agents are less patient and therefore less finicky when trying to locate a potential partner. This leads individuals to try to match with more types of agents and raises the equilibrium knowledge spread.

As each match generates better ideas ( $q_{0}$ higher) individuals choose to meet with more types of agents since the marginal benefit of a diverse knowledge spread is higher. As the scaling factor $\left(s_{0}\right)$ rises, the benefits of effective matches rise. This raises the cost of remaining in relatively less productive interactions, which lowers the equilibrium knowledge spread.

2. Summary of Comparative Statics Results from Section IV.

\begin{tabular}{|c|c|c|c|c|c|c|c|c|}
\hline & $\alpha$ & $\eta$ & $v$ & $A$ & $r$ & $s_{0}$ & $a_{1}$ & $q_{0}$ \\
\hline$\hat{\delta}$ & - & + & + & - & + & + & + & - \\
\hline$\hat{N}$ & + & - & - & + & - & - & - & + \\
\hline
\end{tabular}

This table provides comparative statics results in the following manner. Columns represent exogenous variables, such as $\alpha$. Rows are the endogenous variables. A plus sign means that the associated comparative static, the derivative of the row variable with respect to the column variable, is positive, while a negative sign indicates that this derivative is negative. For instance, the derivative of $\hat{\delta}$ with respect to $\alpha$ 
is negative. The intuition for these results is generally straightforward. A higher arrival rate $(\alpha)$ implies that agents can concentrate on finding more effective opportunities for knowledge exchange and hence will choose a lower knowledge spread $(\hat{\delta}$ lower) -- this is reflected by the shift of the KS locus to the left (see Figure 4). In addition, however, the higher arrival rate raises the gains from migrating because there is less delay between matches. This results in a higher steady-state equilibrium population mass ( $\hat{N}$ higher) which further encourages agents to favor more effective collaborative efforts. The effects of a lower detachment rate are qualitatively the same.

\section{Appendix C: Extensions}

We outline here two extensions of our basic framework. First, we consider an environment to explore the role of transportation costs for information flows. This discussion provides some insights into the findings of Jaffe et al $(1993,1996)$ showing that knowledge spillovers are localized. The second extension attempts to link the patterns of knowledge exchange to an economy's overall measure of diversity. In this way, we discuss why various types of economic clusters such as a city may vary in their degree of specialization. Our results show that economies with the same population size may be either specialized or diverse for the same set of parameters.

\section{The Locational Distance Model}

We demonstrate that the knowledge distance structure in our basic framework can be modified to a locational distance model. We assume agents reside in different locations in the economy and posses different types of knowledge. Unlike the basic framework specified in previous sections, any match would generate the same additional knowledge. Rather, the only cost facing each agent is a transportation cost which is assumed proportional to the distance between the matched agents' residences. Traveling farther for potential collaborators leads a higher flow probability of potential matches, but it leads to a higher cost of transportation via disutility of effort.

There is a continuum of economic agents, labeled by their residence in the city $k \in \mathcal{K}$. Hence, in 
a city with a total population of $N$ (given exogenously), there is a density of $N$ agents at each location. As agents travel through the city, they incur a unit transportation cost, $t$, measured in terms of disutility of effort, for each unit of distance traveled. This cost is incurred in every period of search. Because there are no penalties for differences in types of ideas, each meeting will generate the same additional knowledge and the incremental knowledge enters the production function in the same fashion as in the previous knowledge distance model: $S\left(k, k^{\prime}\right)=q_{0}$. Thus, the asset values of matched $\left(V_{M}\left(\left(k, k^{\prime}\right) ; U\right)\right)$ and unmatched $\left(V_{U}(k ; U)\right)$ agents in this locational distance model are parallel to the Bellman equations in the knowledge distance model:

$$
\begin{gathered}
r V_{M}\left(\left(k, k^{\prime}\right) ; U\right)=y\left(k, k^{\prime}\right)+\eta\left(V_{U}(k ; U)-V_{M}\left(\left(k, k^{\prime}\right) ; U\right)\right) \\
r V_{U}(k ; U)=2 \int_{k}^{k+\delta_{k}}-t d k^{\prime}+\alpha(N) \frac{2}{U} \int_{k}^{k+\delta_{k}}\left(V_{m}\left(\left(k, k^{\prime}\right) ; U\right)-V_{U}(k ; U)\right) U d k^{\prime}
\end{gathered}
$$

where $k-\delta_{k}$ and $k+\delta_{k}$ are the (endogenous) lower and upper bounds, respectively, for locations that an agent $k$ selects to search across for a match. So an agent at location $k$ will search for partners from locations belonging to the set $R(k)=\left[k-\delta_{k}, k+\delta_{k}\right]$, thus determining the location spread. The Bellman equation for unmatched agents reflects that individuals lose utility from traveling across locations in the economy. They incur these transportation costs until they find a partner with whom they can collaborate and share ideas. Thus, once an individual finds a match he no longer incurs the utility loss arising from transportation costs.

For brevity, we concentrate on describing the properties of the steady-state equilibrium in the closed city model as the technical details are identical to our earlier analysis. It is easily shown that higher transportation costs lower welfare in the economy for two reasons. First, as in previous studies, a higher transportation cost (for the same location spread) lowers welfare due to the increased utility loss. Second, this paper argues beyond the conventional work that the transportation cost will induce agents to be more selective when deciding how far to travel searching for partners. As a result, the flow probability of a 
match will be lower and there will be less interaction in the economy, which is again welfare-reducing. Since the existing literature ignores the presence of this latter effect, it is likely that the social costs of transportation or commuting are under-stated.

2. Knowledge Exchange Most Effective when Agents are Alike.

Throughout the paper, we have explored an agent's pattern of interaction with others while acknowledging knowledge exchange depends on differences among agents in terms of types of ideas. In that version of our knowledge distance structure $(\bar{d}>0)$, we assume that the exchange of knowledge is not very effective when agents possess very similar types of knowledge or when agents have little in common.

We now consider an alternative view by assuming knowledge exchange is most effective when agents are alike $(\bar{d}=0)$. Thus, this is a special case of the set of parameters where Assumption 1 does not hold. In general, it can be shown that an agent's unmatched value function is given by:

$$
\begin{aligned}
V_{U}(\delta ; U)= & \left(\frac{4 \alpha(U) \delta_{k}}{r+\eta+4 \alpha(U) \delta_{k}}\right) \frac{A}{r}\left(q_{0}+s_{0} a_{0}-\frac{1}{2} s_{0} a_{1} \delta_{k}\right), \text { where } \delta_{k}<\bar{d} \\
& \left(\frac{2 \alpha(U)\left(\delta_{k}+\bar{d}\right)}{r+\eta+2 \alpha(U)\left(\delta_{k}+\bar{d}\right)}\right) \frac{A}{r}\left(q_{0}+s_{0} a_{0}-\frac{1}{2} s_{0} a_{1}\left[\frac{\bar{d}^{2}+\delta_{k}^{2}}{\bar{d}+\delta_{k}}\right]\right) \text { where } \delta_{k}>\bar{d}
\end{aligned}
$$

For the case where $\bar{d}=0$, the value function is:

$$
V_{U}(\delta ; U)=\left(\frac{2 \alpha(U) \delta_{k}}{r+\eta+2 \alpha(U) \delta_{k}}\right) \frac{A}{r}\left(q_{0}+s_{0} a_{0}-\frac{1}{2} s_{0} a_{1} \delta_{k}\right)
$$

As in Section III, we analyze the determinants of the knowledge spread when the population mass is given exogenously. Although the hypothesis concerning knowledge creation is different than that we pursue in Section III, the underlying determinants of the knowledge spread in the economy remain the same. As 
before, agents choose their selection strategy recognizing tradeoffs between higher probabilities of matches (a contact rate effect) and more effective matches (a knowledge efficacy effect). Because the underlying costs and benefits of matching are the same as in Section III, the properties of the steady-state equilibrium are also similar.

There is an intermediate case where $\bar{d}$ satisfies neither Assumption 1 nor $\bar{d}=0$. This case does not admit a closed-form solution for the relationship between the individual's knowledge spread and the population mass. Our analysis does seem to suggest that, for a given mass of unmatched agents, a higher value of $\bar{d}$ is associated with a smaller knowledge spread. The smaller value of the knowledge spread implies that individuals are more selective of their partners for collaboration which leads to a higher mass of unmatched agents in the steady-state. A higher steady-state mass of unmatched agents further encourages agents to be more selective since the arrival rate of matches is higher when there is a higher mass of unmatched agents in the economy.

\section{Determination of Economy-wide Diversity}

Our final objective is to link patterns of knowledge exchange in an economy to its overall measure of diversity in such a way that we can discuss why various types of economic clusters such as a city may vary in their degree of specialization. We let $b$ represent the circumference of the knowledge space $\boldsymbol{K}$ in the economy (initially set at one) and assume that a local economy (a nation, a region, or a city) will select $b$. We attempt to study the interactions between patterns of knowledge exchange and an economy's overall measure of diversity by envisioning that the economy-wide diversity depends on the economy's average knowledge spread, $b=b(\delta)$, a hypothesis in analogy to the specification of the externality of knowledge/human capital as in Romer (1986) and Lucas (1988). Since our focus is merely on exploring the determinants of an economy's overall measure of diversity, we restrict our attention to examining this setup within the context of a closed-city model as in Section III. 
As before, we derive the knowledge spread of a representative individual. As long as the economy-wide diversity is increasing and sufficiently convex in the average knowledge spread, multiple equilibria emerge for the same set of parameters as a result of self-fulfilling prophecies. We find there exists both a specialized equilibrium and a diversified equilibrium. The specialized equilibrium emerges because when agents believe that the overall economy is very specialized, they expect a higher density of agents across ideas and can thus concentrate on more effective matching. The resultant knowledge spread is therefore more specialized and the economy-wide diversity turns out to be narrow--a self-fulfilling prophecy. By similar arguments, the diversified equilibrium emerges when agents believe that the economy is more diverse. These equilibria are Pareto-rankable as welfare is higher in the specialized equilibrium than in the diversified equilibrium. This provides a role for city planners to induce the economy to select a more specialized set of industries in an effort to help encourage more effective means of informal learning from others. Of course, one cannot directly compare this welfare result with the empirical findings in Glaeser et al (1992) in which a more diversified city is associated with higher employment growth and welfare.

Our conclusion that a specialized equilibrium is Pareto superior depends on the form of knowledge exchange. One could extend our knowledge-distance framework with the optimal level of idea-diversity $(\bar{d})$ to allow for "industries" by partitioning the knowledge space into clusters of types of knowledge. For example, industry $i$ may encompass knowledge types along the interval $\left[i, i+c_{i}\right]$ where $c_{i}$ represents the Lebesgue measure of cluster (or industry) $i$. Because interaction with individuals from the same industries will contain knowledge that is too similar to promote highly effective knowledge exchange, economies with more industrial diversity may have higher welfare since they allow for the possibility to meet with individuals from a greater variety of industries. In this manner, the model would allow for a diversified equilibrium to be associated with more effective knowledge exchange and more rapid economic development as in Glaeser et al. (1992). 


\section{References}

Abdel-Rahman, H. M., 1990, Agglomeration Economies, Types and Sizes of Cities, Journal of Urban Economics, 27, 25-45.

Abdel-Rahman, H. M. and M. Fujita, 1990, Product Variety, Marshallian Externalities and City Sizes, Journal of Regional Science, 30, 165-183.

, and ___ 1993, Specialization and Diversification in a System of Cities, Journal of Urban Economics, 33, 189-222.

Acemoglu, D., 1996, A Microfoundation for Social Increasing Returns in Human Capital Accumulation, Quarterly Journal of Economics, 111, 779-804.

Audretsch, David B., and Maryann P. Feldman, 1996, R\&D Spillovers and the Geography of Innovation and Production, American Economic Review, 86, 630-640.

Benabou, R., 1996, Equity and Efficiency in Human Capital Investment: The Local Connection, Review of Economic Studies, 63, 237-264.

Berliant, M., S. Peng and P. Wang, 1998, Production Externalities and Urban Configurations, mimeo, Washington University - St. Louis.

Black, D., and J.V. Henderson, 1999, A Theory of Urban Growth, Journal of Political Economy, 107, 252-284.

, 1998, Local Human Capital Externalities, Educational Segregation, and Inequality, mimeo, Brown University.

Burdett, K. and M. Coles, 1997, Marriage and Class, Quarterly Journal of Economics, 112, 141-168.

Ciccone, A. and R.E. Hall, 1997, Productivity and the Density of Economic Activity, American Economic Review, 86, 54-70.

Diamond, P.A., 1982, Aggregate Demand Management in Search Equilibrium, Journal of Political Economy, 90, 881-894.

Eaton, J. and Z. Eckstein, 1997, Cities and Growth: Theory and Evidence from France and Japan, Regional Science and Urban Economics, 27, 443-474.

Feldman, Maryann P., 1994, The Geography of Innovation, (Kluwer Academic Publishers, Boston, MA).

Feller, I., 1971, The Urban Location of United States Invention, 1860-1910, Explorations in Economic History, 24, 285-303.

Fujita, M. and F. Ogawa, 1982, Multiple Equilibria and Structural Transition of Non-Monocentric Urban Configurations, Regional Science and Urban Economics, 12, 161-196. 
1989, Urban Economic Theory: Land Use and City size, (Cambridge University Press, New York, NY).

Glaeser, E.L., H.D. Kallal, J.A. Scheinkman, and A. Shleifer, 1992, Growth in Cities, Journal of Political Economy 100, 1126-1152.

, J. Scheinkman, and A. Shleifer, 1995, Economic Growth in a Cross-Section of Cities, Journal of Monetary Economics, 36, 117-143.

, 1997, “Learning in Cities,” NBER Working Paper No. 6271.

Griliches, Z., 1990, Patent Statistics as Economic Indicators: A Survey, Journal of Economic Literature, 28, 1661-1707.

Helsley, R.W. and W.C. Strange, 1990, Matching and Agglomeration Economies in a System of Cities, Journal of Urban Economics, 189-212.

, and __ 1999, Innovation and Input Sharing in Cities, mimeo, University of British

Columbia.

Henderson, J.V., 1974, The Sizes and Types of Cities, American Economic Review, 64, 640-656.

Henderson, J.V., A. Kuncoro and M. Turner, 1995, Industrial Development in Cities, Journal of Political Economy, 103, 1067-1090.

Higgs, 1971, American Inventiveness, 1870-1920, Journal of Political Economy, 79, 661-667.

Ioannides, Y.M., 1994, Product Differentiation and Endogenous Growth in a System of Cities, Regional Science and Urban Economics, 24, 461-484.

Jacobs, J. 1969, The Economy of Cities, (Random House, New York, NY).

Jaffe, A.B., M. Trajtenberg, and R. Henderson, 1993, Geographic Localization of Knowledge Spillovers as Evidenced by Patent Citations, Quarterly Journal of Economics, 108, 577-598.

Jaffe, A.B. and M. Trajtenberg, 1996, "Flows of Knowledge from Universities and Federal Labs: Modeling the Flow of Patent Citations Over Time and Across Institutional and Geographic Boundaries," NBER Working Paper No. 5712.

Jovanovic, B., 1979, Job Matching and the Theory of Turnover, Journal of Political Economy, 87, 972990. and R. Rob, 1989, The Growth and Diffusion of Knowledge, Review of Economic Studies, $56,569-582$.

Kelley, A., 1973, Scale Economies, Inventive Activity, and the Economics of American Population Growth, Explorations in Economic History, 26, 35-52. 
Krugman, P., 1991, Geography and Trade, (MIT Press, Cambridge, MA).

Kuznets, S., 1962, "Population Change and Aggregate Output," in Demographic and Economic Change in Developed Countries (Princeton: Princeton University Press (for NBER)), 324-340.

Laing, D., T. Palivos, and P. Wang, 1995, Learning, Matching, and Growth, Review of Economic Studies, 62, 115-129.

Lucas, R.E., Jr., 1988, On the Mechanics of Economic Development, Journal of Monetary Economics, 22, $3-42$.

Marshall, A., 1895, Principles of Economics, (Macmillan and Co., London).

Palivos, T. and P. Wang, 1997, Spatial Agglomeration and Endogenous Growth, Regional Science and Urban Economics, 26, 645-669.

Pred, A, 1966, The Spatial Dynamics of U.S. Urban-Industrial Growth, 1800-1914 (MIT Press, Cambridge, MA).

Rauch, J., 1993, Productivity Gains from Geographic Concentration of Human Capital: Evidence from Cities, Journal of Urban Economics, 34, 380-400.

Romer, P., 1986, Increasing Returns and Long-Run Growth, Journal of Political Economy, 94, 10021037.

Saxenian, A., 1994, Regional Advantage: Culture and Competition in Silicon Valley and Route 128, (Harvard University Press, Cambridge, MA).

Stigler, G., 1951, The Division of Labor is Limited By the Extent of the Market, Journal of Political Economy, 59, 185-193.

Thompson, W., 1962, "Locational Differences in Inventive Effort and Their Determinants," in R. Nelson(ed.), The Rate and Direction of Inventive Activity: Economic and Social Factors, (Princeton: Princeton University Press (for NBER)), 253-276.

Trejos, A. and R. Wright, 1995, Search, Bargaining, Money, and Prices, Journal of Political Economy, $103,118-141$. 
Figure 1: Knowledge Space of the Economy

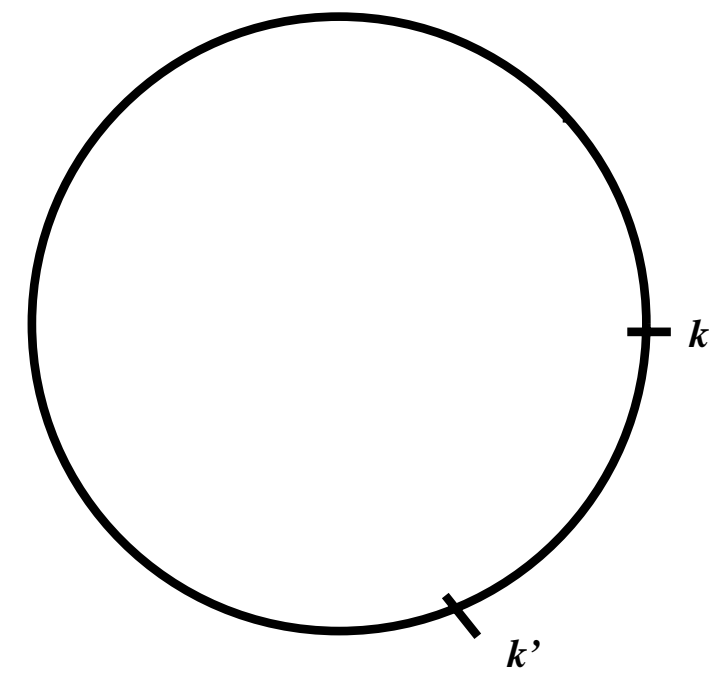

Figure 2: Role of Heterogeneity for Knowledge Creation

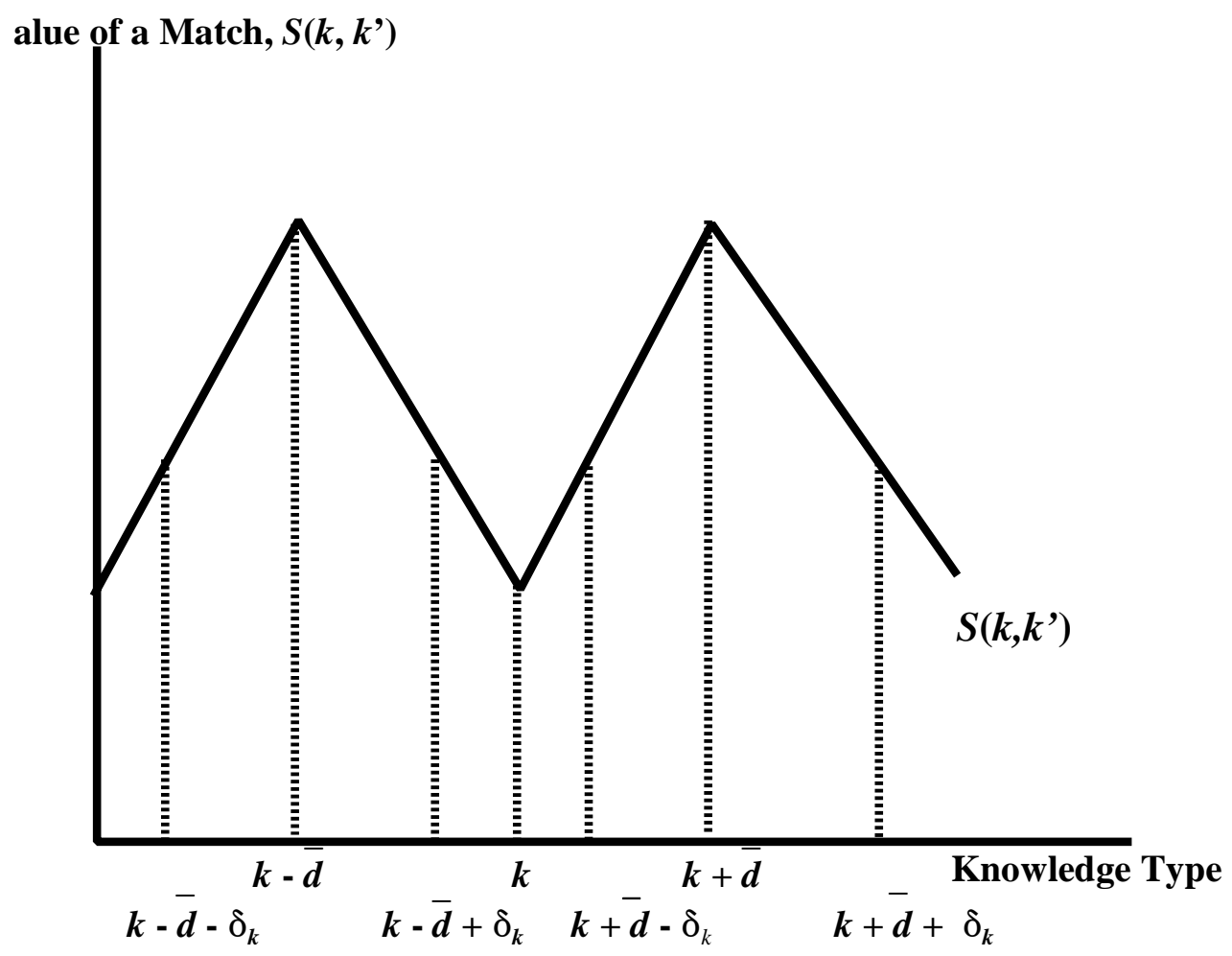


Figure 3: Knowledge Space and Selection of Knowledge Spread

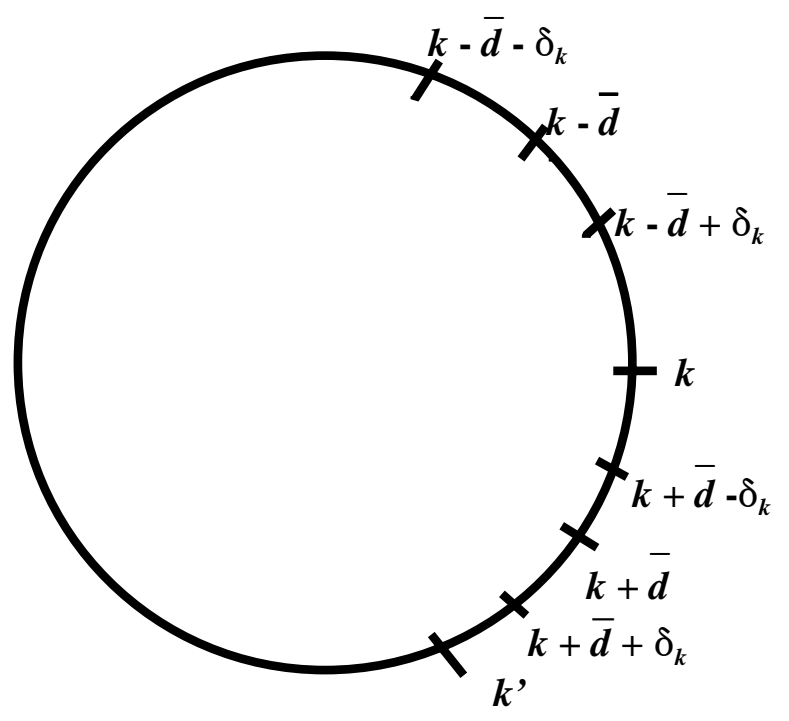

Figure 4: Equilibrium Entry (Migration) Decisions

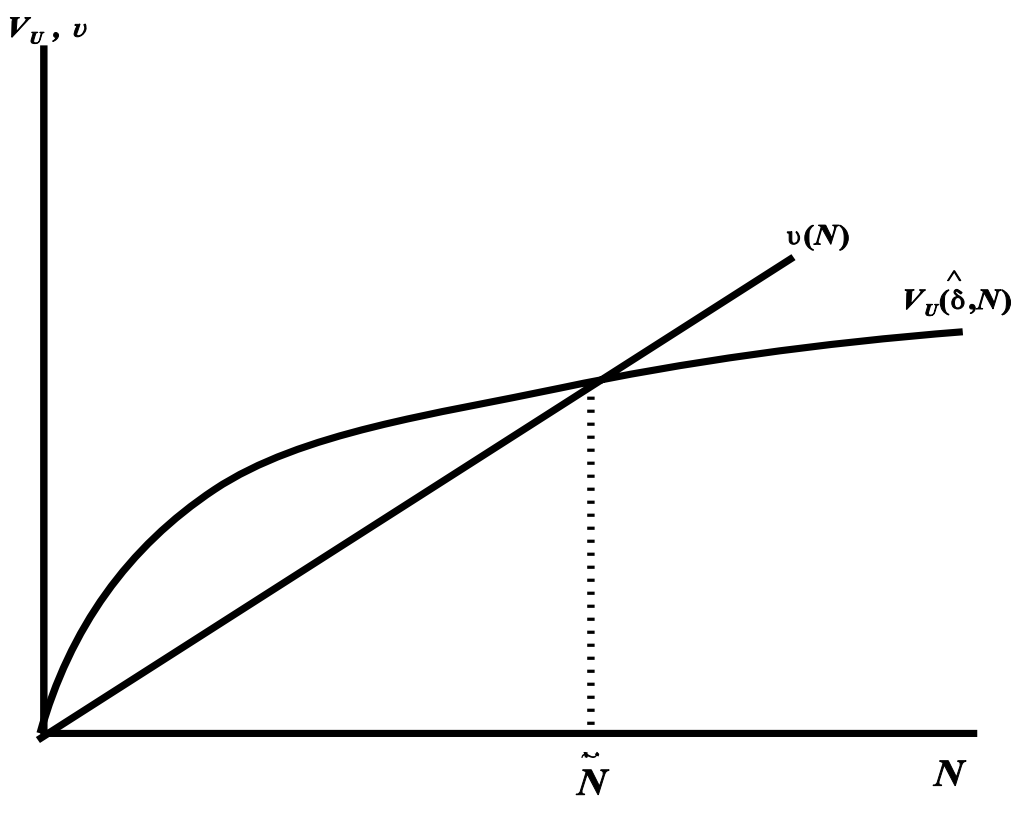


Figure 5: Steady-State Equilibrium under Endogenous Migration $(\alpha(U)=\alpha U)$

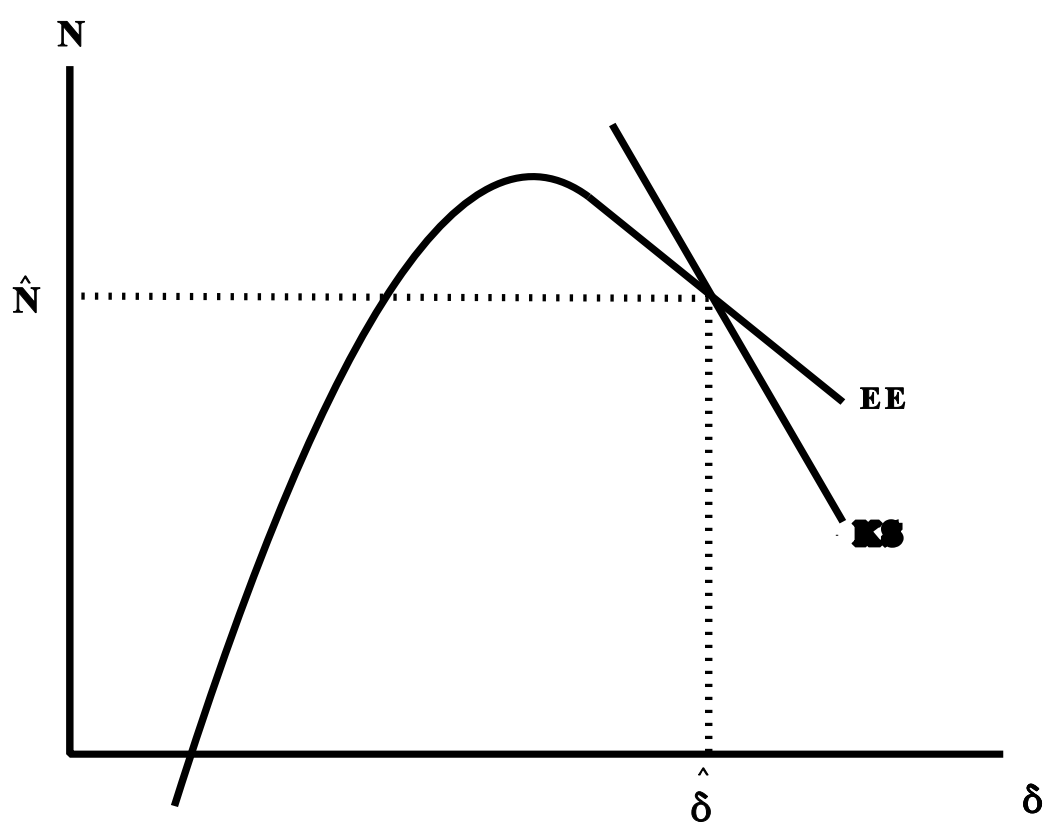

Figure 6: Comparison Between Planner's Solution and Equilibrium when Congestion Externality Dominates

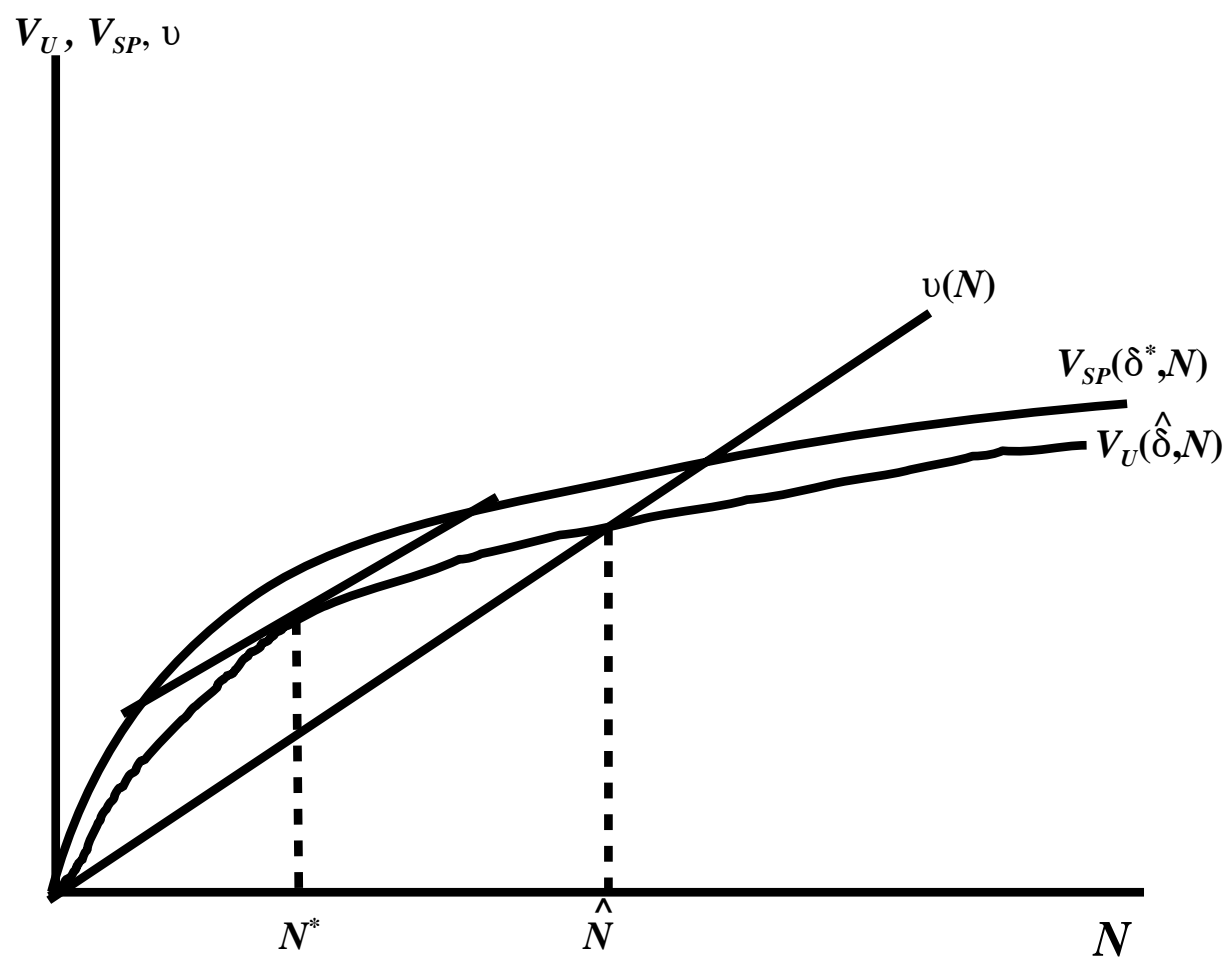


Figure 7: Comparison Between Planner's Solution and Equilibrium when Matching Externality Dominates

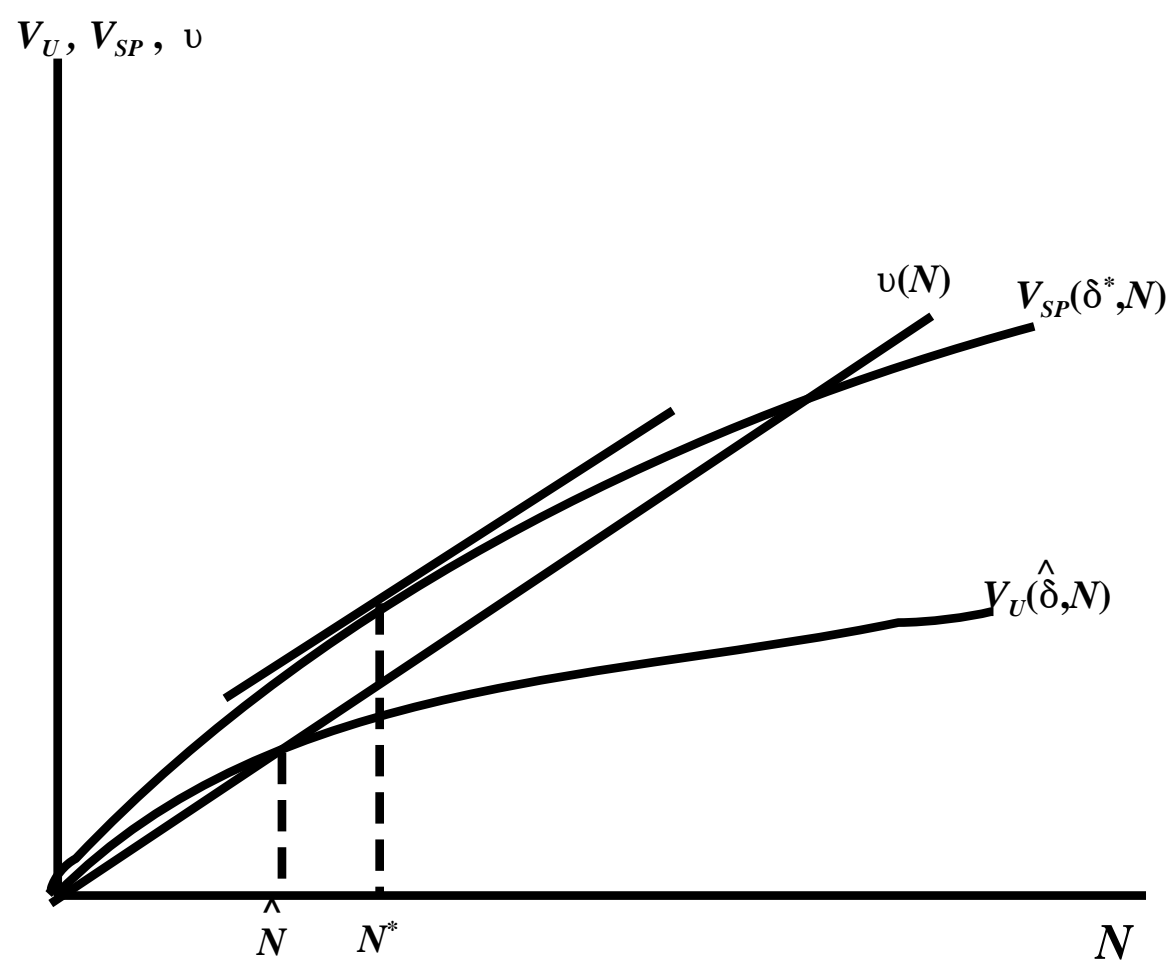

